\title{
Quantitative Characterisation of Surface Defects and Composition on PtRu Nanoparticles Using Aberration-Corrected TEM/STEM
}

\author{
L. Y. Chang ${ }^{1,2}$, S. Lazar ${ }^{1,3}$, E. A. Baranova ${ }^{4}$, C. Bock ${ }^{4}$ and G. A. Botton ${ }^{1}$ \\ 1 Canadian Centre for Electron Microscopy, Dept of Materials Science and Engineering, McMaster \\ University, Hamilton, ON, L8S 4L7, Canada. \\ 2 Monash Centre for Electron Microscopy, Monash University, Vic 3800, Australia. \\ 3 FEI Company, the Netherlands \\ 4 Institute for Chemical Processes and Environmental Technology, National Research Council, \\ Montreal Road, Ottawa, ON, K1A 0R6, Canada
}

The detailed structure and composition on the surfaces of nanoparticles play a crucial role on their catalytic properties. Platinum based nanoparticles have been widely used as electrocatalysts for polymer electrolyte membrane fuel cells and direct methanol fuel cells. The addition of secondary metallic element has improved the efficiency and reduced the $\mathrm{CO}$ poisoning, according to the bifunctional mechanism. Here, the bimetallic PtRu systems have been developed in order to tune the efficiency of fuel cells [1]. These particles can be made using simple colloidal synthesis methods. The size and overall composition can be varied easily and alloy vs. core-shell type particles can be made. Several parameters are of particular importance for these systems. Firstly, the spatial distribution of Pt and $\mathrm{Ru}$ surface sites affect the electronic structure and hence the catalytic properties of these particles. Furthermore, defect structures on the surface can also play a key role, providing e.g., more active nucleation sites for water adsorption, and potentially increase the stability of these catalysts. Hence, there is an essential need to identify the precise surface structure and composition of these catalysts that in turn provides input into synthesis and structure-activity correlation of these systems.

High-Resolution Electron Microscopy (HREM) is a powerful technique to study the morphology and surfaces of nanoparticles [2]. Restoring the phase of the specimen exit wave function from a through focal series [3] gives an aberration free, complex exit wave function. The improved signal to noise ratio in the restored phase makes direct imaging of atoms on surfaces of nanoparticles possible [4-5]. On the other hand, high-angle annular dark field (HAADF) STEM gives Z contrast imaging with high spatial resolution, making it suitable for measuring atomic column by column compositional distribution of bimetallic nanoparticles.

Figure 1a shows the phase of a PtRu nanoparticle restored from a focal series acquired with a Titan 80-300 Cubed located at the Canadian Centre for Electron Microscopy. Figure 1b shows the excellent match of the simulated phase based on the model that gives the best fit to the experimental phase. The individual atomic column in the restored phase of this nanoparticle is well resolved, and their atomic positions were measured using a least-square fitting procedure, allowing us to extract the information of surface atom deviation. The intensity distribution of individual atomic column of the HAADF STEM image of a PtRu nanoparticle, as shown in Figure 2(a), gives the information of composition distribution of $\mathrm{Pt}$ and $\mathrm{Ru}$. The large fluctuation of the intensity distributions, shown in Figure 2(b) can only be attributed to the mixed $\mathrm{Pt}$ and $\mathrm{Ru}$ atomic columns in the particle. Comparisons of intensity distribution profiles with those of pure Pt nanoparticles and the detailed multislice [6] simulations will give carry out in order to extract quantitative composition information for the bimetallic nanoparticles. 


\section{References}

[1] E. Baranova, C. Bock, D. Ilin, D. Wang, B. MacDougall, Surface Science, 2006, 600 (17), 3502-3511

[2] L. Marks et al., Nature 303 (1983) 316.

[3] R. R. Meyer et al., Ultramicroscopy 92 (2002) 89.

[4] L. Gontard-Cervera et al., Angewandte Chemie Int. Ed. 46 (2007) 3683.

[5] G. Lovely et al., Appl. Phys. Lett. 88 (2006) 093124.

[6] J. M. Cowley and A. F. M. Moodie, Acta. Crystallogr. 10 (1957) 609.
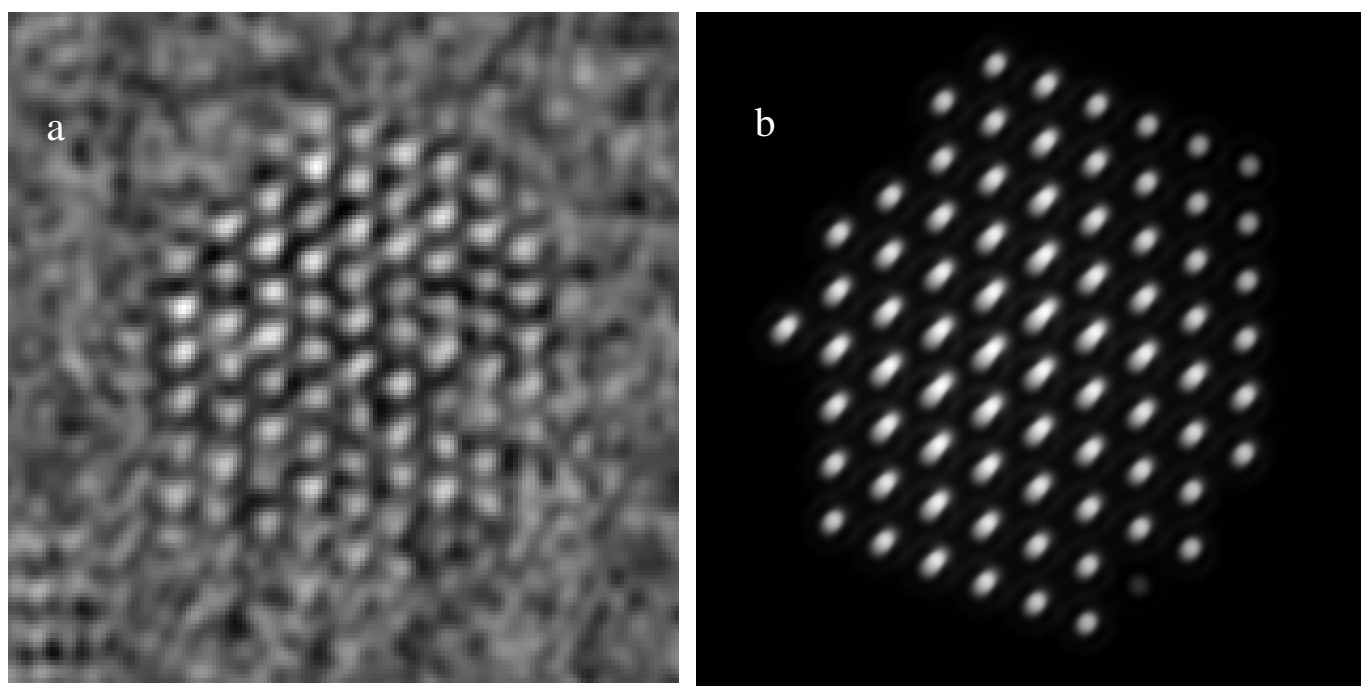

FIG 1. (a) Phase of a PtRu nanoparticle exit wave function restored from a focal series taken from the aberration-correced Titan 80-300 Cubed (b) Simulated phase from an atomic model based on (a).
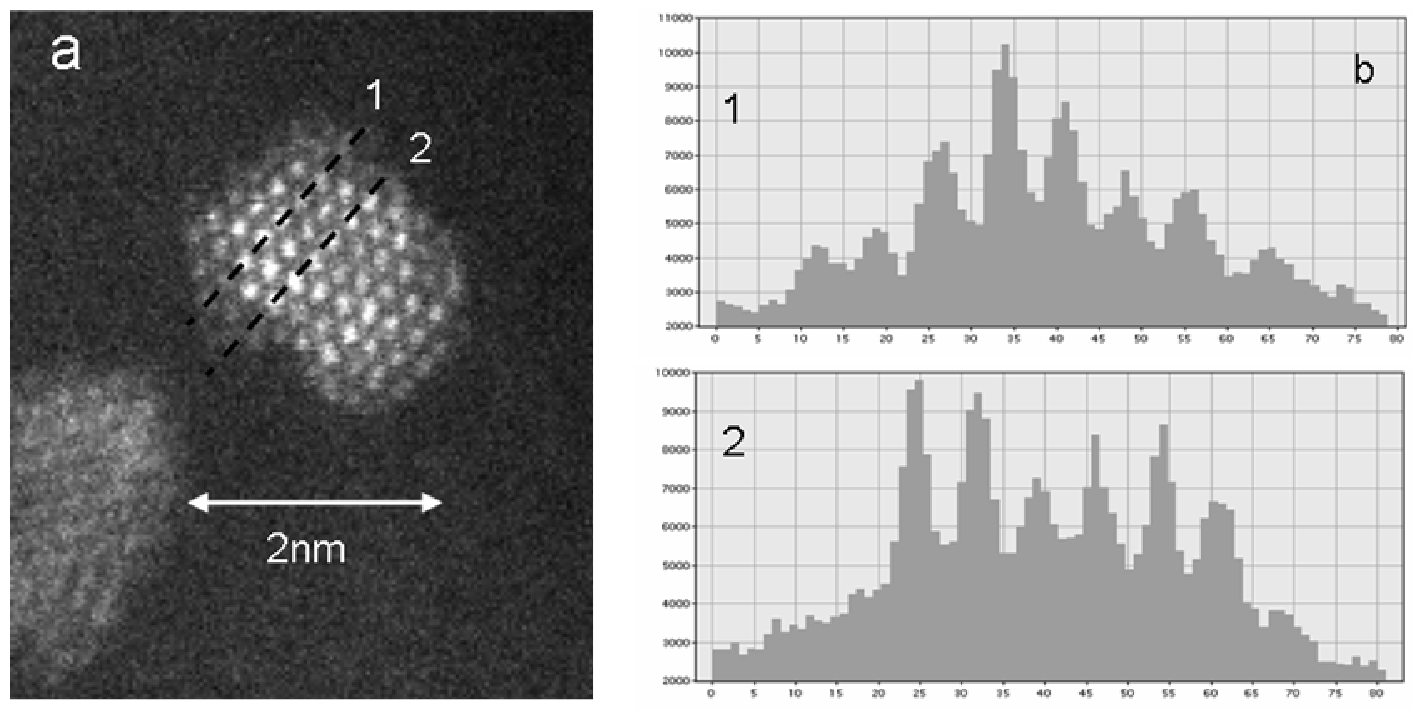

FIG. 2. (a) HAADF STEM image of a PtRu nanoparticle. (b) linescans of HAADF STEM intensities taken from the profiles shown in (a). 